\title{
Looking Through the Vascular Normalization Window: Timing Antiangiogenic Treatment and Chemotherapy with 99m Tc-Annexin A5
}

\begin{abstract}
$\mathbf{O}$
ver the last 100 years, it has become well established that tumors have a rich network of vascular growth and undergo angiogenesis readily (1). In 1971, Judah Folkman posited that molecular factors could be identified that promote angiogenesis (2) and drive the formation of abnormal tumor blood vessels. His groundbreaking hypothesis was that if these factors could be inhibited, vessels would regress, and the tumor would starve to death. As a result of Folkman's revolutionary ideas, research in the last 40 years has resulted in several antiangiogenic drugs entering the practice of medical oncology.
\end{abstract}

See page 1786

The rationale behind antiangiogenic therapy is that such treatment would cause the degeneration of tumor vasculature that is heterogeneous and highly abnormal at the macroscopic and microscopic levels. Tumor vessels tend to be dilated, tortuous, distorted, and disorganized. Furthermore, within tumors there are areas of high vascular density and others with low vascular density, as well as loss of diameter control and shunting. Endothelial cell junctions are loose, poorly connected,

Received Sep. 27, 2011; revision accepted Oct. 4, 2011.

For correspondence or reprints contact: Michael R. Lewis, Department of Veterinary Medicine and Surgery College of Veterinary Medicine, 900 E. Campus Dr., University of Missouri Columbia, Columbia, MO 65211.

E-mail: LewisMic@missouri.edu

Published online Oct. 12, 2011.

COPYRIGHT ( 2011 by the Society of Nuclear Medicine, Inc.

DOI: 10.2967/jnumed.111.097725 or overlapping, and perivascular cells (pericytes and vascular smooth muscle cells) surrounding the endothelium are also abnormal, causing vessel leakage.

One of the major players in causing these adverse processes is vascular endothelial growth factor A (VEGFA; more commonly known as VEGF). VEGF was found by Ferrara and others to be a potent angiogenic mitogen $(3,4)$. By binding to VEGF receptor 2, VEGF plays an indispensible role in mediating angiogenic activity in vascular endothelial cells. Moreover, VEGF overexpression leads to the formation of neovasculature with the structurally abnormal morphology characteristic of tumors.

Taken together, the unwanted characteristics of abnormal angiogenesis play a major role in impeding the delivery of cytotoxic drugs and other systemic treatments, such as biologic agents. As a consequence, the idea of antiangiogenic therapy was developed with the rationale that regression of blood vessels would cause tumors to become dormant. In 1993, Ferrara et al. showed that treatment with an antiVEGF antibody reduced vasculature in and inhibited the growth of several tumor types xenografted into nude mice (5). Ferrara's group also demonstrated that anti-VEGF therapy resulted in subcutaneous xenograft growth delay and reduction in liver metastasis in a mouse model of colon cancer (6).

Despite these promising preclinical results, the outcomes of anti-VEGF antibody treatment alone in patients have been disappointing. Poor objective response rates and lack of improvement in overall survival have been characteristic of anti-VEGF treatment. In 1 study, administration of bevacizumab, an anti-VEGF antibody, to colorectal cancer patients pretreated with chemotherapy resulted in an overall response rate of only $3.3 \%$ (7). However, randomized phase III trials of bevacizumab plus conventional chemotherapy have shown improvements in overall survival and time to tumor progression when compared with chemotherapy alone, with evidence of a synergistic effect. In a large number of phase II-IV trials treating metastatic colorectal cancer, the first-line efficacy of bevacizumab in combination with irinotecan-based chemotherapy have generally resulted in progression-free survival intervals of 9-14 mo and overall survivals of 1928 mo $(8,9)$. What seems counterintuitive about these clinical results, however, is that inhibition of VEGF should result in vascular regression. Yet at the same time, VEGF inhibition improves the effectiveness of chemotherapy, on which efficient drug delivery depends.

In 2001, Jain proposed the vascular normalization hypothesis $(10,11)$. This hypothesis states that, instead of merely effecting regression of vasculature, careful use of antiangiogenic therapy may cause the grossly abnormal structure and function of tumor blood vessels to return to a more normal state. The implications of this idea are the possibilities that tumor growth could be controlled, hypoxia could be reduced, and the efficacy of systemic therapies could be improved. An important mechanism for vascular normalization is inhibition of VEGF signaling, as it is a critical factor controlling proliferation and survival of endothelial cells. However, when vascular normalization is induced, it is transient, characterized by a time 
window of 1-2 d, in which tumor cell kill by cytotoxic agents is markedly increased. A critical factor, though, is the timing of cancer therapy, to take advantage of this transient normalization.

In this issue of The Journal of $\mathrm{Nu}$ clear Medicine, Vangestel et al. (12) used $99 \mathrm{~m}$ Tc-tricarbonyl His-annexin A5 ( ${ }^{99 m}$ Tc-His-annexin A5) microSPECT to probe the vascular normalization window. In a nude mouse model of colon cancer, they administered sequential treatments of bevacizumab to normalize vasculature and irinotecan to induce apoptosis. Radiolabeled annexin A5 has been used to detect apoptosis after single-dose chemotherapy in tumor-bearing rats $(13,14)$. In addition, Vangestel's group previously validated the use of ${ }^{99 \mathrm{~m}} \mathrm{Tc}$ tricarbonyl His-annexin A5 for imaging tumor apoptosis in vivo during single-dose irinotecan and bevacizumab monotherapies, respectively (15).

The present studies of Vangestel et al. explored the timing between bevacizumab and irinotecan that would produce the most effective killing of colon cancer cells. Bevacizumab had some profound effects on colon tumors shortly after administration. These effects were generally most notable on days 2 and 4 after bevacizumab. Pericyte coverage increased, the vessel maturity index increased dramatically, and VEGF-A concentrations decreased in the tumor. At day 4, microvessel density decreased to the point that it was significantly lower than saline controls. However, by day 6 the vessel maturity index decreased dramatically and some mature vessels were likely degenerating, suggesting that the vascular normalization window had begun to close.

After characterizing the effects of bevacizumab in the murine colon cancer model, Vangestel et al. investigated tumor uptake of ${ }^{99 \mathrm{~m}} \mathrm{Tc}$-annexin A5 at $12-48 \mathrm{~h}$ after a single administration of irinotecan. In all experiments, tumor accumulation of ${ }^{99 \mathrm{~m}} \mathrm{Tc}$-annexin A5 correlated with caspase-3 activity, confirming that apoptosis was indeed the mechanism of cell death. A peak of tumor uptake and image intensity occurred $48 \mathrm{~h}$ after irinotecan and $2 \mathrm{~d}$ after bevacizumab treatment, compared with saline controls. However, a considerably greater uptake and image intensity occurred at $4 \mathrm{~d}$ after bevacizumab and $24 \mathrm{~h}$ after irinotecan administration. By $6 \mathrm{~d}$ after bevacizu$\mathrm{mab}$ and $48 \mathrm{~h}$ after irinotecan, only a small difference in tumor uptake and image intensity was observed, again suggesting that the vasculature normalization window was closing. The best results were obtained $4 \mathrm{~d}$ after bevacizumab and $24 \mathrm{~h}$ after irinotecan. Although vessel maturity index increased by day 2 , tumor hypoxia also increased relative to day 4 , reducing the effectiveness of chemotherapy. Altogether, it appeared that the optimal timing of this dual-therapy regimen was to administer bevacizumab $4 \mathrm{~d}$ before irinotecan, inducing a more rapid onset of maximum apoptosis. Furthermore, the highest uptake of ${ }^{99 m}$ Tc-annexin A5 in the dual-therapy regimen was about $60 \%-70 \%$ higher than that for bevacizumab monotherapy, indicative of a synergistic effect of the 2 drugs.

Interestingly, the findings of Vangestel et al. fit nicely with the 1 - to $2-d$ window of vascular normalization predicted by the Jain hypothesis. Understanding the duration of this window is critical to administering effective chemotherapy in combination with antiangiogenic drugs. Measurement of the normalization window by noninvasive imaging of chemotherapy-induced apoptosis could be a powerful tool for clinicians to make decisions regarding timing of pretreatment with drugs designed to return the vasculature to a more normal state. Such approaches could optimize delivery of commonly used chemotherapeutics. More important, perhaps, is that the development of new treatment modalities, not limited to chemotherapy, might be greatly aided by noninvasive imaging of apoptosis.

Implications of the results obtained by Vangestel et al. may reach far beyond the detection of vascular normalization and apoptosis in chemotherapy. For example, clinical use of cancer immunotherapy has remained a considerable challenge to date. Synergistic effects have been observed in tumor-bearing mouse models of antiVEGF therapy plus immunotherapy, given by adoptive cell transfer (16) or use of cancer vaccines (17). In one study (16), a schedule-dependent improvement in efficacy was observed when the antibody was given $2 \mathrm{~d}$ before adoptive cell transfer. This result suggests that an open normalization time window might increase T-cell delivery into tumors. Because such therapies are capable of inducing tumor cell apoptosis, ${ }^{99 \mathrm{~m}} \mathrm{Tc}$-annexin A5 imaging may shed light on the nature of tumor vasculature normalization during immunotherapy. Whether the treatment be chemotherapy, radiotherapy, immunotherapy, or a combination thereof, noninvasive imaging of apoptosis may allow us to act while the window is open.

\section{Michael R. Lewis \\ University of Missouri \\ Harry S. Truman Memorial Veterans' \\ Hospital \\ Columbia, Missouri}

\section{REFERENCES}

1. Goel S, Duda DG, Xu L, et al. Normalization of the vasculature for treatment of cancer and other diseases. Physiol Rev. 2011;91:1071-1121.

2. Folkman J. Tumor angiogenesis: therapeutic implications. N Engl J Med. 1971;285:1182-1186.

3. Keck PJ, Hauser SD, Krivi G, et al. Vascular permeability factor, an endothelial cell mitogen related to PDGF. Science. 1989;246:1309-1312.

4. Leung DW, Cachianes G, Kuang WJ, Goeddel DV, Ferrara N. Vascular endothelial growth factor is a secreted angiogenic mitogen. Science. 1989;246: 1306-1309.

5. Kim KJ, Li B, Winer J, et al. Inhibition of vascular endothelial growth factor-induced angiogenesis suppresses tumor growth in vivo. Nature. 1993; 362:841-844.

6. Warren RS, Yuan H, Matli MR, Gillett NA, Ferrara N. Regulation by vascular endothelial growth factor of human colon cancer tumorigenesis in a mouse model of experimental liver metastasis. J Clin Invest. 1995;95:1789-1797.

7. Giantonio BJ, Catalano PJ, Meropol NJ, et al. Bevacizumab in combination with oxaliplatin, fluorouracil, and leucovorin (FOLFOX4) for previously treated metastatic colorectal cancer: results from the European Cooperative Oncology Group Study E3200. J Clin Oncol. 2007;25:15391544 .

8. Heinemann V, Hoff PM. Bevacizumab plus irinotecan-based regimens in the treatment of metastatic colorectal cancer. Oncology. 2010;79: 118-128. 
9. Saltz LB, Lenz HJ, Kindler HL, et al. Randomized phase II trial of cetuximab, bevacizumab, and irinotecan compared with cetuximab and bevacizumab alone in irinotecan-refractory colorectal cancer: the BOND-2 study. J Clin Oncol. 2007;25:4557-4561.

10. Jain RK. Normalizing tumor vasculature with anti-angiogenic therapy: a new paradigm for combination therapy. Nat Med. 2001;7:987-989.

11. Jain RK. Normalization of tumor vasculature: an emerging concept in antiangiogenic therapy. Science. 2005;307:58-62.

12. Vangestel C, Van de Wiele C, Van Damme N, et al. ${ }^{99 \mathrm{~m} T c-t r i c a r b o n y l ~ H i s-a n n e x i n ~ A 5 ~ m i c r o-S P E C T ~}$ demonstrates increased cell death by irinotecan during the vascular normalization window caused by bevacizumab. J Nucl Med. 2011;52:17861794.

13. Mochizuki T, Kuge Y, Zhao S, et al. Detection of apoptotic tumor response in vivo after a single dose of chemotherapy with ${ }^{99 \mathrm{~m}} \mathrm{Tc}$-annexin V. $\mathrm{J} \mathrm{Nucl}$ Med. 2003;44:92-97.

14. Takei T, Kuge Y, Zhao S, et al. Time course of apoptotic tumor response after a single dose of chemotherapy: comparison with ${ }^{99 \mathrm{~m}} \mathrm{Tc}$-annexin $\mathrm{V}$ uptake and histologic findings in an experimental model. J Nucl Med. 2004;45:2083-2087.

15. Vangestel C, Van de Wiele C, Mees G, et al. SPECT imaging of the early time course of therapy-induced cell death using ${ }^{99 \mathrm{~m}} \mathrm{Tc}-(\mathrm{CO}) 3$ His-annexin A5 in a colorectal cancer xenograft model. Mol Imaging. In press.

16. Shrimali RK, Yu Z, Theoret MR, Chinnasamy D, Restifo NP, Rosenberg SA. Antiangiogenic agents can increase lymphocyte infiltration into tumor and enhance the effectiveness of adoptive immunotherapy of cancer. Cancer Res. 2010;70: 6171-6180.

17. Manning EA, Ullman JGM, Leatherman JM, et al. A vascular endothelial growth factor receptor-2 inhibitor enhances antitumor immunity through an immune-based mechanism. Clin Cancer Res. 2007;13:3951-3959. 